\title{
A prospective study of treatments for adult-onset divergence insufficiency-type esotropia
}

Eric R. Crouch, MD, ${ }^{a}$ Trevano W. Dean, MPH, ${ }^{b}$ Raymond T. Kraker, MSPH, ${ }^{b}$ Aaron M. Miller, MD, MBA, ${ }^{c}$ Courtney L. Kraus, MD, ${ }^{d}$ Kammi B. Gunton, MD ${ }^{e}$ Michael X. Repka, MD, MBA, ${ }^{d}$ Justin D. Marsh, MD, ${ }^{f}$ Monte A. Del Monte, MD, ${ }^{g}$ Paula A. Luke, OD, ${ }^{h}$ Jason H. Peragallo, MD, ${ }^{i}$ Katherine A. Lee, MD, PhD, ${ }^{j}$ Maynard B. Wheeler, MD, ${ }^{k}$ Timothy J. Daley, MD ${ }^{l}$ David K. Wallace, MD, MPH, ${ }^{m}$ Susan A. Cotter, OD, MS, ${ }^{n}$ Jonathan M. Holmes, BM, BCh, ${ }^{\circ}$ on behalf of the Pediatric Eye Disease Investigator Group*

Author affiliations: ${ }^{a}$ Virginia Pediatric Eye Center, Virginia Beach, Virginia; ${ }^{b}$ Jaeb Center for Health Research, Tampa, Florida; ${ }^{c}$ Houston Eye Associates, The Woodlands, Texas; ${ }^{d}$ Wilmer Eye Institute, Baltimore, Maryland; ${ }^{e}$ Wills Eye Hospital, Philadelphia, Pennsylvania; ${ }^{f}$ Children's Mercy Hospitals and Clinics, Kansas City, Missouri; ${ }^{g}$ WK Kellogg Eye Center, University of Michigan, Ann Arbor; ${ }^{h}$ Pacific University College of Optometry, Portland, Oregon; ${ }^{i}$ Emory Eye Center, Atlanta, Georgia; ${ }^{j}$ St. Luke's Hospital, Boise, Idaho; ${ }^{k}$ Concord Ophthalmologic Associates, Concord, New Hampshire; 'Medical Associates Clinic, Dubuque, Iowa; ${ }^{m}$ Indiana University School of Medicine, Indianapolis; ${ }^{n}$ Southern California College of Optometry at Marshall B. Ketchum University, Fullerton, California; ${ }^{\circ}$ University of Arizona, Tucson

*A full list of participating members of the Pediatric Eye Disease Investigator Group is included as eSupplement 1 (available at jaapos.org).

Funding support: National Eye Institute of National Institutes of Health, Department of Health and Human Services, EY011751, EY023198, and EY018810, EY024333. The funding organization had no role in the design or conduct of this research.

Presented at the 45th Annual Meeting of the American Association for Pediatric Ophthalmology and Strabismus, San Diego, California, March 27-31, 2019.

Submitted October 15, 2020.

Revision accepted February 24, 2021.

Correspondence: Eric R. Crouch, MD, c/o Jaeb Center for Health Research, 15310 Amberly Drive, Suite 350, Tampa, FL 33647 (email: ercrouch@gmail.com).

Word count: 2,923

Abstract only: 242

This is the author's manuscript of the article published in final edited form as:

Crouch, E. R., Dean, T. W., Kraker, R. T., Miller, A. M., Kraus, C. L., Gunton, K. B., Repka, M. X., Marsh, J. D., Del Monte, M. A., Luke, P. A., Peragallo, J. H., Lee, K. A., Wheeler, M. B., Daley, T. J., Wallace, D. K., Cotter, S. A., \& Holmes, J. M. (2021). A prospective study of treatments for adult-onset divergence insufficiency-type esotropia. Journal of American Association for Pediatric Ophthalmology and Strabismus, 25(4), 203.e1-203.e11. https://doi.org/10.1016/j.jaapos.2021.02.014 


\begin{abstract}
Purpose

To describe 10-week and 12-month outcomes following treatment for divergence insufficiencytype esotropia in adults.

\section{Methods}

In this prospective observational study, 110 adults with divergence insufficiency-type esotropia, with a distance esodeviation measuring $2^{\Delta}$ to $30^{\Delta}$ and at least $25 \%$ larger at distance than near, and binocular diplopia present at least "sometimes" at distance, were enrolled at 28 sites when initiating new treatment. Surgery, prism, or divergence exercises/therapy were chosen at the investigator's discretion. Diplopia was assessed at enrollment and at 10-week and 12-month outcome examinations using a standardized diplopia questionnaire (DQ). Success was defined as DQ responses of "rarely" or "never" when looking straight ahead in the distance, with no alternative treatment initiated.

Results
\end{abstract}

Of the 110 participants, 32 (29\%) were prescribed base-out prism; none had received prior treatment for esotropia. Success criteria were met by 22 of 30 at 10 weeks $(73 \%$; 95\% CI, 54\%$88 \%)$ and by 16 of 26 at 12 months (62\%; 95\% CI, 41\%-80\%). For the $76(68 \%)$ who underwent strabismus surgery ( $82 \%$ of whom had been previously treated with prism), success criteria were met by 69 of 74 at 10 weeks (93\%; 95\% CI, 85\%-98\%) and by 57 of 72 at 12 months (79\%; 95\% CI, 68\%-88\%).

\title{
Conclusions
}

In this study cohort, both base-out prism as initial therapy and strabismus surgery (usually following prism) were successful in treating diplopia for most adults with divergence 
insufficiency-type esotropia when assessed during the first year of follow-up. 
Divergence insufficiency-type (DI-type) esotropia is a common form of adult strabismus, ${ }^{1}$ accounting for $10 \%$ of all cases of new-onset adult strabismus, with an incidence of 6.0 per 100,000 , and a higher incidence in elderly adults in population based-studies. ${ }^{2}$ DI-type esotropia, also known as age-related distance esotropia, is often defined as an acquired comitant esotropia where the angle of deviation is greater at distance than at near. ${ }^{2}$ The etiology of DI-type esotropia in adults remains unclear. Hypotheses include age-related degeneration of the orbital connective tissues, commonly referred to as "sagging eye syndrome"3 and shortening or increased tone of the medial rectus muscles. ${ }^{4,5}$

A variety of treatments are commonly used to address DI-type esotropia, including prism correction, ${ }^{6-9}$ divergence exercises/therapy,${ }^{10}$ and strabismus surgery. ${ }^{5,11-13}$ Surgical approaches include lateral rectus resections ${ }^{12,14,15}$ and medial rectus recessions, either in combination or singly. ${ }^{5,11,13}$ Most previous reports of DI-type esotropia in adults are limited by retrospective design, non-standardized follow-up schedule, and non-standardized data collection. The current study was designed to prospectively describe clinical characteristics of adults with DI-type esotropia, the frequency of specific treatments, and 10-week and 12-month treatment outcomes. Treatment was not standardized in the present study because one aim was to determine the frequency of specific treatments across a large group of eye care providers.

\section{Subjects and Methods}

This study was supported through a cooperative agreement with the National Eye Institute of the National Institutes of Health and was conducted according to the Declaration of Helsinki by the Pediatric Eye Disease Investigator Group (PEDIG) at academic and private practice clinical sites. The study protocol complied with the US Health Insurance Portability and Accountability Act of 1996. Informed consent forms were approved by institutional review boards, and written 
consent was obtained from each participant. The study is listed on www.clinicaltrials.gov (NCT02510040), and the full study protocol and procedures manual are available on the PEDIG website (www.pedig.net).

\section{Eligibility and Enrollment}

Adults $>18$ years of age diagnosed with DI-type esotropia were eligible if they had a distance esodeviation of $2^{\Delta}-30^{\Delta}$ that was at least $25 \%$ larger than at near, measured by the prism and alternate cover test (PACT), and diplopia at least "sometimes" in the distance. Any coexisting vertical deviation had to be less than the distance esodeviation and $\leq 10^{\Delta}$ by PACT. Additional eligibility criteria are in Table 1.

A standardized diplopia questionnaire (DQ), ${ }^{16}$ was used to assess diplopia in specific gaze positions (reading, distance straight ahead, right, left, up, down, any other) and the frequency for each position of gaze ("never," "rarely," "sometimes," "often," or "always"). For eligibility, participants needed to report diplopia on the DQ with a frequency of "sometimes," "often," or "always" in straight-ahead gaze at distance fixation during the week prior to enrollment. At enrollment, all participants were asked to complete the DQ as though they were wearing their current refractive correction without prism. Patients who were already wearing prism and not experiencing diplopia in prism, could be enrolled for surgical treatment if they reported diplopia on the DQ as "sometimes," "often," or "always" when not wearing prism for straight-ahead gaze at distance fixation. All participants completed the Adult Strabismus (AS-20) questionnaire, ${ }^{17}$ which evaluates health-related quality of life (HRQOL). Medical history was extracted from the medical record.

Participants were only enrolled when a new treatment for their DI-type esotropia was being initiated by the investigator (either prism, surgery, or divergence exercises/therapy). 
Participants were not eligible if strabismus surgery had been performed prior to enrollment, or, for those to be treated with prism or divergence exercises/therapy, if they had received the same treatment within the prior year. In contrast, those undergoing surgery were allowed to have undergone treatment with prism or divergence exercises/therapy. Choice and specifics of treatment (eg, surgical method, dosage, prism magnitude, exercise specifics) were at investigator discretion. The treatment cohorts were essentially distinct. One participant was included in both prism and surgery cohorts, because the participant was originally enrolled into prism treatment, but failed at 10 weeks and was then reenrolled as a participant treated with surgery.

If treatment changed after enrollment (eg, exercises/therapy to prism, or prism to surgery), an early outcome examination was completed. If the change was to surgery (not including a reoperation) and the participant still met eligibility criteria, the examination served both as outcome examination for the initial treatment (eg, prism or exercises/therapy) and enrollment examination for surgical treatment.

\section{Follow-up Testing Procedures and Data Collection}

Enrolled participants were scheduled to return $10( \pm 3)$ weeks and $12( \pm 2)$ months following initiation of their new treatment. For 10-week and 12-month outcome assessments, participants were asked to complete DQ in their habitual refractive correction, whether that correction included prism. As such, responses at 10-weeks and 12-months for prism group reflected their status while wearing prism correction. The only exception to completing the DQ in prism correction was the infrequent scenario where those prescribed surgery or exercises/therapy failed those treatments and were wearing prism at a follow-up visit. In order to appropriately represent specific types of surgical failure, participants who were wearing prism at the 10-week and/or 12month outcome examination, but not originally prescribed prism at enrollment, were instructed 
to complete the DQ as though not wearing prism. This exception only affected $9(12 \%)$ of 76 surgery participants who completed 10-week or 12-month outcomes. Changes in treatment type or intensity were also documented.

\section{Primary Outcome Measures}

At each follow-up visit, treatment was classified as successful if the participant indicated on DQ diplopia was "rarely" or "never" over the preceding week, when looking straight ahead at a distance fixation target. Treatment was classified as "failed" if the participant indicated diplopia was present "always," "often," or "sometimes" on DQ when looking straight ahead at distance fixation.

Treatment was classified as "failed" if an alternative treatment modality (different from enrollment) had been started, with the exception of using temporary therapeutic prism or exercises during the immediate postoperative period for surgical patients, prior to 10 weeks. Using prism or exercises/therapy beyond 10 weeks from strabismus surgery was considered a failure of surgical treatment. For primary analysis of success, changes in prism magnitude for participants treated with prism at enrollment, was not considered as failure.

Determination of success or failure at 12 months was assessed independently of 10-week outcomes.

\section{Secondary Outcome Measures}

We evaluated two prespecified secondary outcomes. The DQ score ${ }^{16}$ is an established quantitative measure of diplopia severity (range 0-100; not diplopic to always diplopic in all fields of gaze) and AS-20, ${ }^{17,18}$ an established quality-of-life measure. The AS-20 ${ }^{17,18}$ has four unidimensional scores (Reading Function, General Function, Self-Perception, Interactions), with Rasch-scoring used for each domain and rescaling from 0 (worst HRQOL) to 100 (best HRQOL) 
using published look-up tables. ${ }^{18,19}$

In a post hoc secondary analysis, diplopia status when reading was incorporated into success criteria, with secondary success outcome defined as diplopia "rarely" or "never" for reading and distance straight ahead. Not all participants reported diplopia with reading at enrollment; $42(38 \%)$ never had diplopia in the reading position and 21 (19\%) reported rarely having diplopia. For these participants, who reported no or rare reading diplopia at enrollment, success at distance straight ahead gaze was sufficient for secondary success.

\section{Statistical Analyses}

For primary and secondary definitions of success, participants who met success criteria were tabulated according to treatment modality, for both overall cohort and within subgroups, and corresponding exact $95 \%$ confidence intervals calculated. The mean, standard deviation, and mean change from enrollment to each outcome visit and 95\% CI were calculated for DQ scores and AS-20 domain scores.

Subgroups of prism treatment were defined post-hoc, in the following arbitrary categories: correcting ( $\geq 100 \%$ of the esodeviation, measured by PACT at distance), high relieving (99\% to $60 \%)$, and low relieving $(<60 \%)$. Because this subgroup analysis evaluated effectiveness of prescribed prism, failure was also declared if prism strength was increased.

Subgroups of surgical treatment were defined a priori as (1) bilateral medial rectus muscle recession, (2) bilateral lateral rectus muscle resection, and (3) other. Success rates with surgery overall were analyzed by whether prism had been prescribed prior to surgery.

\section{Results}

\section{Treatment Prescribed at Enrollment and Enrollment Characteristics}

Between September 2015 and December 2017, 110 participants were enrolled at 28 sites. Three 
(3\%) were prescribed divergence exercises/therapy, 32 (29\%) were prescribed base-out prism, and $76(68 \%)$ were prescribed surgery. One participant was included in both prism and surgery cohorts, because the participant was originally enrolled with prism, but failed prism at 10 weeks and was then reenrolled with surgery. Given that few participants received exercises/therapy, the results are limited to prism and surgery, although summary results are included in the tables.

Enrolled participants were primarily white (96\%) and female (67\%), with a median age of 71.2 years (range, 18.8-90.8). Compared with participants who received prism, surgery participants were more likely to have received previous treatment; $82 \%$ had previous prism treatment. Participants treated with surgery reported more severe diplopia in the distance (based on DQ score) and larger deviations at enrollment (based on PACT). Additional data on demographics, medical history, and clinical characteristics are presented in Tables 2 and 3.

\section{Outcomes with Prism}

For 32 participants prescribed base-out prism, the primary success criteria at distance were met in 22 of 30 at 10 weeks (73\%; 95\% CI, 54\%-88\%) and 16 of 26 at 12 months (62\%; 95\% CI, 41\%-80\%). See Table 4. One participant was considered to have failed prism treatment at 12 months because surgery was prescribed at the 10-week visit. Follow-up was completed by $78 \%$ of prism participants (Figure 1). For the secondary definition of success including both reading gaze and distance straight-ahead gaze, success proportions and 95\% CIs were identical to the primary definition of success. Regarding prism type, $47 \%$ were prescribed ground-in prism, and 53\% press-on Fresnel prism at enrollment. At 10 weeks, 57\% originally treated with prism were wearing ground-in prism whereas $43 \%$ wore Fresnel prism. At 12 months, $72 \%$ originally treated with prism were wearing ground-in prism whereas $16 \%$ wore Fresnel prism, and 12\% no prism.

Mean DQ scores and mean AS-20 general function domain scores improved at 10 weeks 
and 12 months (Table 5).

Treatment success and secondary outcomes by prism type at enrollment (correcting, high relieving, or low relieving) are shown in Table 6A and 6B. All three approaches were associated with improved DQ scores at 10 weeks and 12 months. AS-20 general function domain scores improved from enrollment to 10 weeks. There were no marked differences in DQ scores or AS20 domain scores between prism approaches.

\section{Outcomes with Strabismus Surgery}

For the 76 who underwent strabismus surgery, primary success criterion at distance was met in 69 of $74(93 \% ; 95 \% \mathrm{CI}, 85 \%-98 \%)$ at 10 weeks and 57 of $72(79 \% ; 95 \% \mathrm{CI}, 68 \%-88 \%)$ at 12 months (Table 4). Follow-up was completed in 71 of 76 patients $(93 \%)$ who underwent surgery (Figure 1). For the secondary definition of success including both reading gaze and distance straight-ahead gaze, 67 of 74 (91\%; 95\% CI, 81\%-96\%) were successful at 10 weeks and 56 of $72(78 \% ; 95 \% \mathrm{CI}, 66 \%-87 \%)$ at 12 months.

Mean DQ scores and mean AS-20 general function and reading domain scores improved at 10 weeks and 12 months. In addition, self-perception and interaction domain scores also improved at 10 weeks and 12 months (Table 5).

Treatment success by the two most frequently performed types of surgery, bilateral medial rectus muscle recession and bilateral lateral rectus muscle resection, are given in Table 7A. The small number of participants who underwent bilateral lateral rectus muscle resections precluded formal statistical comparisons. No striking differences in DQ scores or AS-20 domain scores existed between two main surgical groups (Table 7B).

No significant differences between treatment success were found irrespective of preoperative prism, but small sample size precludes formal comparison (Table 8). 


\section{Discussion}

Within our investigator group, the most common treatments for DI-type esotropia in adults were strabismus surgery (68\%) and base-out prism glasses (29\%). Success rates were acceptable at 12 months following initiation of treatment; $79 \%$ (95\% CI, 68\%-88\%) with surgery and 62\% (95\% $\mathrm{CI}=41 \%$ to $80 \%$ ) with prism. We did not compare success rates between surgery and prism cohorts, because none of the prism-treated participants had received prior treatment for DItype esotropia, and $82 \%$ of surgical participants had previously received prism.

Other case series have also reported high success rates of prism, ${ }^{6-9}$ and surgery, ${ }^{5,11,13-15}$ for the treatment of DI-type esotropia in adults. Previous studies have not incorporated standardized outcome measures along with a prospective study design. Interestingly, two-thirds of our participants were female, and this possible predisposition has been reported by others. ${ }^{4,20}$

The success rate with prism is predictably high since small angle esodeviations are commonly treated with prism, and appropriately prescribed prism should eliminate diplopia. We did not standardize the method of prescribing prism, and the amount of prism prescribed ranged from low relieving to fully correcting relative to distance esodeviation. Although we did not find large differences in treatment success between the three prism approaches, our sample sizes for these subgroups were small, limiting comparisons.

We are unaware of previous studies using HRQOL as an outcome measure for adult DItype esotropia. The AS-20 was designed as a patient-reported outcome measure across the spectrum of strabismus conditions. ${ }^{17}$ Because adults with DI-type esotropia most often have small to moderate esodeviations, we would not expect subnormal scores on the psychosocial domains of self-perception or interactions. Enrollment scores in our study indicated minimal impact. In contrast, subnormal scores ${ }^{21}$ were found in the general function and reading function 
domains for more severe, larger-magnitude near deviations. In these functional domains we found marked improvement following treatment.

The most common surgery performed among investigators was bilateral medial rectus muscle recessions. One concern regarding this surgical procedure for distance esotropia is that the surgery might induce an exodeviation at near, and diplopia at near. Nevertheless, we found success by secondary criteria (never or rare diplopia at both distance and near) was almost identical to that by the primary definition (never or rare diplopia at distance only). Induced symptomatic exodeviation at near was rare in our study as has been reported by others, ${ }^{13}$ though follow-up was limited to 12 months. We had hoped to compare outcomes between different surgical procedures (a priori) and between different prism prescribing strategies (post hoc), but small subgroups precluded statistical analysis. Additionally, surgical success rates reflect the surgical doses chosen by specific investigators. We did not study strategies to improve outcomes such as preoperative prism adaptation or increasing surgical dose.

Regarding whether previous treatment with prism influences subsequent success with surgery, we had an insufficient number of participants undergoing surgery without previous prism treatment to formally evaluate. It is possible that fully correcting esodeviations with baseout prism might decrease divergence amplitudes over time and reduce surgical success. Alternatively, partially correcting esodeviations, and allowing better motor and sensory fusion, might result in increased divergence amplitudes and increase the likelihood surgery would be successful. The question of prior prism effect on surgical outcome is worthy of further study.

Our study has limitations. We did not have untreated controls and cannot comment on the natural history of this condition. We also did not randomize treatment allocation. Treatment assignment was at investigator discretion, with possible allocation bias. None of the participants 
treated with prism had received prior treatment for their esotropia, whereas $82 \%$ of participants undergoing surgery had previously been treated with prism. Furthermore, participants treated with surgery had more severe diplopia at distance fixation and larger esodeviations than those treated with prism. Although we had excellent retention for surgery (93\% at 12 months), we had lower retention for prism ( $78 \%$ at 12 months). It is unclear whether less than optimal follow-up created bias, because participants might have sought alternative treatment elsewhere or might have been more prone to return with symptoms. In addition, we did not standardize the prescription of prism or the type of strabismus surgery; standardized doses of treatment might have yielded different results. Long-term Fresnel prism, in a small proportion of participants, may have influenced our results, particularly in quality-of-life scores. Finally, when dichotomizing a continuous or ordinal measure, such as 5-level DQ responses, there is risk of misclassification, reported as up to $20 \% .^{22}$ 


\section{References}

1. Goseki T, Suh SY, Robbins L, Pineles SL, Velez FG, Demer JL. Prevalence of sagging eye syndrome in adults with binocular diplopia. Am J Ophthalmol 2020;209:55-61.

2. Martinez-Thompson JM, Diehl NN, Holmes JM, Mohney BG. Incidence, types, and lifetime risk of adult-onset strabismus. Ophthalmology 2014;121:877-82.

3. Rutar T, Demer JL. "Heavy Eye" syndrome in the absence of high myopia: a connective tissue degeneration in elderly strabismic patients. J AAPOS 2009;13:36-44.

4. Ridley-Lane M, Lane E, Yeager LB, Brooks SE. Adult-onset chronic divergence insufficiency esotropia: clinical features and response to surgery. J AAPOS 2016;20:11720.

5. Bothun ED, Archer SM. Bilateral medial rectus muscle recession for divergence insufficiency pattern esotropia. J AAPOS 2005;9:3-6.

6. Scheiman M, Gallaway M, Ciner E. Divergence insufficiency: characteristics, diagnosis, and treatment. Am J Optom Physiol Opt 1986;63:425-31.

7. Tamhankar MA, Ying GS, Volpe NJ. Effectiveness of prisms in the management of diplopia in patients due to diverse etiologies. J Pediatr Ophthalmol Strabismus 2012;49:222-8.

8. Prangen Ade H, Koch FL. Divergence insufficiency: a clinical study. Trans Am Ophthalmol Soc 1937;35:136-48.

9. Godts D, Mathysen DG. Distance esotropia in the elderly. Br J Ophthalmol 2013;97:1415-19.

10. Caloroso EE, Rouse MW, Cotter SA. Clinical management of strabismus. Boston: Butterworth-Heinemann; 1993. 
11. Thomas AH. Divergence insufficiency. J AAPOS 2000;4:359-61.

12. Stager DR, Sr., Black T, Felius J. Unilateral lateral rectus resection for horizontal diplopia in adults with divergence insufficiency. Graefes Arch Clin Exp Ophthalmol 2013;251:1641-4.

13. Chaudhuri Z, Demer JL. Medial rectus recession is as effective as lateral rectus resection in divergence paralysis esotropia. Arch Ophthalmol 2012;130:1280-84.7

14. Wiggins RE, Jr., Baumgartner S. Diagnosis and management of divergence weakness in adults. Ophthalmology 1999;106:1353-6.

15. Simpson GV. Primary divergence insufficiency. Trans Am Ophthalmol Soc 1973;71:152161; discussions 161-2.

16. Holmes JM, Liebermann L, Hatt SR, Smith SJ, Leske DA. Quantifying diplopia with a questionnaire. Ophthalmology 2013;120:1492-6.

17. Hatt SR, Leske DA, Bradley EA, Cole SR, Holmes JM. Development of a quality-of-life questionnaire for adults with strabismus. Ophthalmology 2009;116:139-44.e135.

18. Leske DA, Hatt SR, Liebermann L, Holmes JM. Evaluation of the Adult Strabismus-20 (AS-20) questionnaire using Rasch analysis. Invest Ophthalmol Vis Sci 2012;53:263039.

19. Leske DA, Hatt SR, Liebermann L, Holmes JM. Lookup tables versus stacked Rasch analysis in comparing pre- and postintervention Adult Strabismus-20 data. Transl Vis Sci Technol 2016;5:11.

20. Repka MX, Downing E. Characteristics and surgical results in patients with age-related divergence insufficiency esotropia. J AAPOS 2014;18:370-73.

21. Hatt SR, Leske DA, Liebermann L, Holmes JM. Incorporating health-related quality of 
life into the assessment of outcome following strabismus surgery. Am J Ophthalmol 2016;164:1-5.

22. Mansukhani SA, Hatt SR, Leske DA, Holmes JM. Test-retest reliability of the revised diplopia questionnaire. J AAPOS 2019;23:319.e1-5. 
Table 1. Eligibility criteria met by all patients enrolled in the study

1. Age $\geq 18$ years

2. Adult-onset divergence-type esotropia (at $\geq 18$ years of age)

3. No prior strabismus surgery

4. Symptoms of diplopia at distance with a frequency of "sometimes," "often," or "always" as measured by the diplopia questionnaire

5. Distance esodeviation of $2^{\Delta}$ to $30^{\Delta}$ and distance deviation 1.25 times (25\% larger than) near deviation by prism and alternate cover test (PACT)

6. $\quad$ No more than $5^{\Delta}$ difference between right and left gaze by PACT

7. No more than $10^{\Delta}$ difference between the primary position at distance and either upgaze or downgaze $\leq 10^{\Delta}$ by PACT

8. Any coexisting vertical deviation must be less than distance esodeviation and $\leq 10 \Delta$ by prism and alternating cover test

9. Visual acuity $20 / 50$ or better in both eyes by ETDRS or Snellen

10. No paralytic strabismus (eg, 3rd, 4th, or 6th cranial nerve palsies, skew deviation, Duane syndrome)

11. No restrictive strabismus (eg, blowout fracture, thyroid eye disease, post scleral buckle, Brown syndrome)

12. No monocular diplopia

13. No paretic strabismus, thyroid eye disease, myasthenia gravis, chronic progressive external ophthalmoplegia, or eye movement abnormalities associated with known neurological disease; patients with Parkinson's disease enrolled if nonparetic deviation

14. No inferior or superior oblique overaction defined as $2+$ or greater

15. Ability to acquire single vision while viewing a $20 / 50$ single optotype at $6 \mathrm{~m}$, with or without the aid of prism

16. Ability to understand and complete a survey

17. Investigator is initiating treatment with prism, divergence exercises/therapy, or surgery

18. If initiating treatment with botulinum toxin or surgery, planned injection or surgery to be within 60 days of enrollment

19. Single treatment modality planned (eg, no combined prism and divergence exercises/therapy)

20. Treatment to be initiated has not been used within past 1 year 
Table 2. Baseline characteristics and medical history for all enrolled participants by treatment initiated at enrollment ${ }^{\mathrm{a}}$

\begin{tabular}{|c|c|c|c|c|}
\hline Study parameter & $\begin{array}{l}\text { Prism } \\
(\mathrm{n}=32)\end{array}$ & $\begin{array}{l}\text { Surgery } \\
(\mathrm{n}=76)\end{array}$ & $\begin{array}{c}\text { Divergence } \\
\text { exercises/ } \\
\text { therapy } \\
(\mathrm{n}=3)\end{array}$ & $\begin{array}{l}\text { All participants }{ }^{\mathrm{b}} \\
(\mathrm{N}=111)\end{array}$ \\
\hline Female & $18(56)$ & $55(72)$ & $1(33)$ & $74(67)$ \\
\hline Race/ethnicity: white & $29(91)$ & $76(100)$ & $2(67)$ & $107(96)$ \\
\hline \multicolumn{5}{|l|}{ Age, years } \\
\hline $18-24$ & $0(0)$ & $2(3)$ & $0(0)$ & $2(2)$ \\
\hline 25-34 & $1(3)$ & $1(1)$ & $0(0)$ & $2(2)$ \\
\hline $35-44$ & $1(3)$ & $3(4)$ & $0(0)$ & $4(4)$ \\
\hline $45-54$ & $\begin{array}{l}3 \\
9)\end{array}$ & $7(9)$ & $0(0)$ & $10(9)$ \\
\hline $55-64$ & $6(19)$ & $12(16)$ & $0(0)$ & $18(16)$ \\
\hline $65-74$ & $11(34)$ & $31(41)$ & $2(67)$ & $44(40)$ \\
\hline 75-84 & $8(25)$ & $17(22)$ & $1(33)$ & $26(23)$ \\
\hline 85-91 & $2(6)$ & $3(4)$ & $0(0)$ & $5(5)$ \\
\hline Mean \pm SD & $68.0 \pm 13.8$ & $67.0 \pm 13.9$ & $72.2 \pm 6.4$ & $67.4 \pm 13.7$ \\
\hline Median (IQR) & $69.9(60.5-77.1)$ & $70.9(60.7-75.4)$ & $71.9(66.0-78.8)$ & $71.2(60.8-75.7)$ \\
\hline & \multicolumn{3}{|c|}{ Prior treatment for strabismus ${ }^{c}$} & $100(90)$ \\
\hline Divergence exercises/therapy & $0(0)$ & $7(9)$ & $0(0)$ & $7(6)$ \\
\hline Prism & $0(0)$ & $62(82)$ & $1(33)$ & $63(57)$ \\
\hline None & $32(100)$ & $13(17)$ & $2(67)$ & $47(42)$ \\
\hline \multicolumn{5}{|l|}{ Coexisting neurological conditions } \\
\hline Stroke & $0(0)$ & $3(4)$ & $0(0)$ & $3(3)$ \\
\hline Intracranial tumor & $0(0)$ & $1(1)$ & $0(0)$ & $1(<1)$ \\
\hline \multicolumn{5}{|l|}{ Other conditions } \\
\hline Epiretinal membrane & $0(0)$ & $1(1)$ & $1(33)$ & $2(2)$ \\
\hline Age-related macular degeneration & $1(3)$ & $2(3)$ & $0(0)$ & $3(3)$ \\
\hline Other macular pathology & $1(3)$ & $1(1)$ & $0(0)$ & $2(2)$ \\
\hline Heart disease & $5(16)$ & 7 (9) & $0(0)$ & $12(11)$ \\
\hline Diabetes & $3(9)$ & $5(7)$ & $0(0)$ & $8(7)$ \\
\hline Autoimmune disease & 1 (3) & $1(1)$ & $0(0)$ & 2 (2) \\
\hline Hypertension & $4(13)$ & $10(13)$ & $0(0)$ & $14(13)$ \\
\hline Cancer of the bladder, breast, or prostate & $2(6)$ & $4(5)$ & $0(0)$ & $6(5)$ \\
\hline Other major medical conditions & $5(16)$ & $17(22)$ & $0(0)$ & $22(20)$ \\
\hline
\end{tabular}

$I Q R$, interquartile range; $S D$, standard deviation.

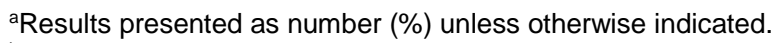

${ }^{\mathrm{b}}$ One participant initially treated with prism was reenrolled after surgery was prescribed at the 10-week visit.

${ }^{\mathrm{c} P a r t i c i p a n t s ~ m a y ~ h a v e ~ r e c e i v e d ~ m o r e ~ t h a n ~ o n e ~ t y p e ~ o f ~ t r e a t m e n t ~ p r i o r ~ t o ~ e n r o l l m e n t . ~}$ 
Table 3. Diplopia and clinical characteristics at enrollment for all enrolled participants according to treatment initiated at enrollment ${ }^{\mathrm{a}}$

\begin{tabular}{|c|c|c|c|c|}
\hline Study parameter & $\begin{array}{c}\text { Prism } \\
(\mathrm{n}=32)\end{array}$ & $\begin{array}{l}\text { Surgery } \\
(n=76)\end{array}$ & $\begin{array}{c}\text { Divergence } \\
\text { exercises/ } \\
\text { therapy } \\
(n=3)\end{array}$ & $\begin{array}{c}\text { All participants } \\
(\mathrm{N}=111)\end{array}$ \\
\hline \multicolumn{5}{|c|}{ Frequency of diplopia at distance during last week } \\
\hline Always & $7(22)$ & $47(62)$ & $2(67)$ & $56(50)$ \\
\hline Often & $11(34)$ & $22(29)$ & $0(0)$ & $33(30)$ \\
\hline Sometimes & $14(44)$ & $7(9)$ & $1(33)$ & $22(20)$ \\
\hline \multicolumn{5}{|l|}{ Frequency of diplopia at near during last week } \\
\hline Always & $1(3)$ & $14(18)$ & $0(0)$ & $15(14)$ \\
\hline Often & $2(6)$ & $10(13)$ & $1(33)$ & $13(12)$ \\
\hline Sometimes & $4(13)$ & $16(21)$ & $0(0)$ & $20(18)$ \\
\hline Rarely & $6(19)$ & $15(20)$ & $0(0)$ & 21 (19) \\
\hline Never & $19(59)$ & $21(28)$ & $2(67)$ & $42(38)$ \\
\hline \multicolumn{5}{|l|}{ Horizontal deviation at distance by PACT, PD } \\
\hline 1-9 esodeviation & $19(59)$ & $6(8)$ & $2(67)$ & $27(24)$ \\
\hline 10-14 esodeviation & $8(25)$ & $29(38)$ & $1(33)$ & $38(34)$ \\
\hline 15-18 esodeviation & $2(6)$ & $17(22)$ & $0(0)$ & $19(17)$ \\
\hline $20-25$ esodeviation & $3(9)$ & $20(26)$ & $0(0)$ & $23(21)$ \\
\hline 26-30 esodeviation & $0(0)$ & $4(5)$ & $0(0)$ & $4(4)$ \\
\hline Mean \pm SD & $9(6)$ & $17(6)$ & $8(6)$ & $14(7)$ \\
\hline Median (IQR) & 8 (4 to 12$)$ & $16(14$ to 20$)$ & 7 (3 to 14$)$ & $14(10$ to 18$)$ \\
\hline \multicolumn{5}{|l|}{ Horizontal deviation at near by PACT, PD } \\
\hline 1-9 exodeviation & $2(6)$ & $1(1)$ & $2(67)$ & $5(5)$ \\
\hline No deviation (orthophoria) & $14(44)$ & $12(16)$ & $0(0)$ & $26(23)$ \\
\hline 1-9 esodeviation & $12(38)$ & $42(55)$ & $1(33)$ & $55(50)$ \\
\hline 10-14 esodeviation & $4(13)$ & $15(20)$ & $0(0)$ & $19(17)$ \\
\hline $15-18$ esodeviation & $0(0)$ & $2(3)$ & $0(0)$ & $2(2)$ \\
\hline $20-25$ esodeviation & $0(0)$ & $4(5)$ & $0(0)$ & $4(4)$ \\
\hline $26-30$ esodeviation & $0(0)$ & $0(0)$ & $0(0)$ & $0(0)$ \\
\hline Mean $\pm S D^{b}$ & $3(4)$ & $7(5)$ & $0(2)$ & $5(5)$ \\
\hline Median $(\mathrm{IQR})^{\mathrm{b}}$ & $1(0$ to 6$)$ & $6(2$ to 10$)$ & $-1(-2$ to 2$)$ & $5(0$ to 8$)$ \\
\hline \multicolumn{5}{|c|}{ Prism needed to fuse in free space for distance viewing ${ }^{\mathrm{C}}$} \\
\hline Horizontal only & $23(79)$ & $61(85)$ & $1(33)$ & $85(82)$ \\
\hline Vertical only & $0(0)$ & $1(1)$ & $0(0)$ & $1(1)$ \\
\hline Both horizontal and vertical & $1(3)$ & $6(8)$ & $1(33)$ & $8(8)$ \\
\hline None & $5(17)$ & $4(6)$ & $1(33)$ & $10(10)$ \\
\hline \multicolumn{5}{|l|}{ Diplopia Questionnaire Score ${ }^{d}$} \\
\hline Mean \pm SD & $41(19)$ & $65(21)$ & $52(34)$ & $58(23)$ \\
\hline Median (IQR) & 39 (24 to 58$)$ & 60 (49 to 84$)$ & $40(25$ to 90$)$ & $57(41-73)$ \\
\hline \multicolumn{5}{|c|}{ Adult Strabismus QOL General Function score } \\
\hline Mean \pm SD & $64(26)$ & $61(21)$ & $65(5)$ & $62(22)$ \\
\hline Median (IQR) & 71 (39 to 84$)$ & 60 (47 to 76$)$ & $68(60$ to 68$)$ & 60 (43 to 76$)$ \\
\hline \multicolumn{5}{|c|}{ Adult-Strabismus QOL Reading Function score ${ }^{e}$} \\
\hline Mean \pm SD & $79(21)$ & $67(24)$ & $90(10)$ & $71(23)$ \\
\hline Median (IQR) & 87 (69 to 95$)$ & 69 (49 to 89) & 89 (79 to 100$)$ & 75 (55 to 90$)$ \\
\hline \multicolumn{5}{|l|}{ Adult-Strabismus QOL Self-perception score } \\
\hline Mean \pm SD & $92(17)$ & $85(20)$ & $93(12)$ & $87(19)$ \\
\hline Median (IQR) & $100(93$ to 100$)$ & 96 (79 to 100$)$ & $100(79$ to 100$)$ & 96 (82 to 100$)$ \\
\hline \multicolumn{5}{|l|}{ Adult-Strabismus QOL interaction score ${ }^{\mathrm{e}}$} \\
\hline Mean \pm SD & $95 \pm 10$ & $94 \pm 12$ & $91 \pm 15$ & $94 \pm 11$ \\
\hline Median (IQR) & $100(97-100)$ & $100(90-100)$ & $100(74-100)$ & $100(90-100)$ \\
\hline
\end{tabular}

$I Q R$, interquartile range; $P A C T$, prism and alternate cover test; $P D$, prism diopters; $Q O L$, quality of life; $S D$, standard deviation.

${ }^{a}$ Results presented as number (\%) unless otherwise indicated.

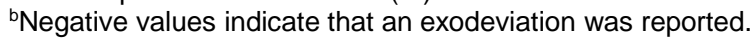

'Data not available for 3 treated with prism and 4 treated with surgery.

${ }^{\mathrm{d}} \mathrm{DQ}$ xcores range from 0 to 100 , with 0 being best (no diplopia) and 100 being worst (diplopia in all gazes all the time).

${ }^{\mathrm{e}} \mathrm{AS}-20$ domain scores range from 0 to 100 , with 0 being the worst HRQOL and 100 being the best HRQOL. 
Table 4. Treatment success

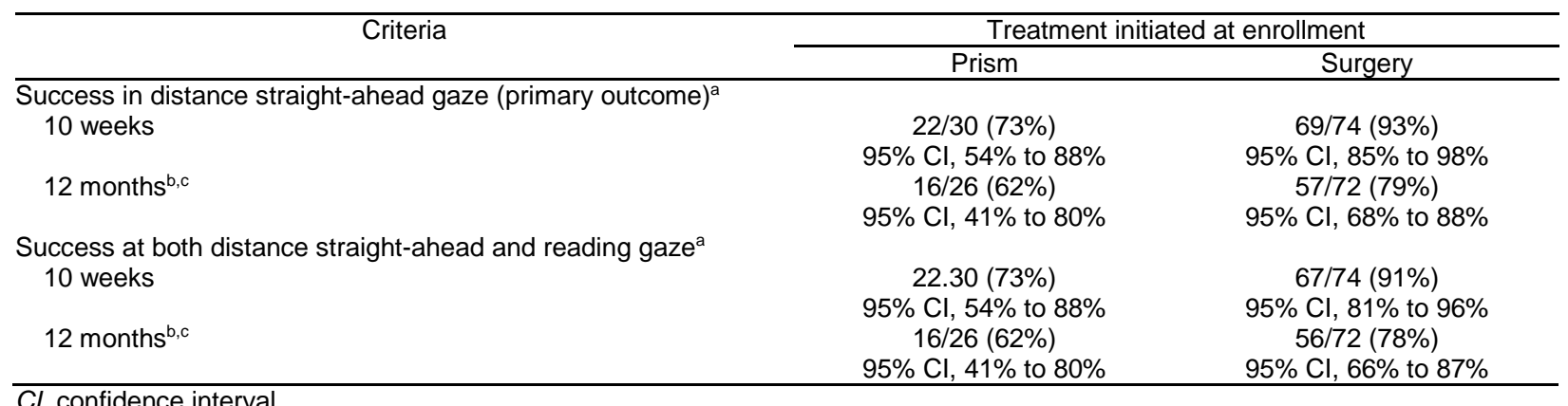

aDefinition of success: report on Diplopia Questionnaire of "rarely" or "never" diplopia over the preceding week and no alternative intervention.

${ }^{b}$ One participant who received prism at enrollment met failure criteria at the 12-month examination despite not completing the 12month examination because surgery was prescribed at the 10 -week examination.

${ }^{c}$ Nine participants who received surgery at enrollment met failure criteria at the 12-month examination because additional treatment was prescribed at or after the 10-week examination, but prior to the 12-month examination; 8 were prescribed prism glasses, and 1 received surgery. 
Table 5. Secondary clinical outcomes

\begin{tabular}{|c|c|c|c|c|c|c|c|c|c|c|}
\hline & \multicolumn{2}{|c|}{ Enrollment } & \multicolumn{4}{|c|}{10 Weeks } & \multicolumn{4}{|c|}{12 Months } \\
\hline & $\begin{array}{c}\text { Prism } \\
(\mathrm{n}=32)\end{array}$ & $\begin{array}{l}\text { Surgery } \\
(\mathrm{n}=76)\end{array}$ & & $\begin{array}{l}\text { Prism } \\
N=30\end{array}$ & & $\begin{array}{l}\text { Surgery }{ }^{\mathrm{a}} \\
(\mathrm{n}=74)\end{array}$ & & $\begin{array}{l}\text { Prism } \\
(\mathrm{n}=25)\end{array}$ & & $\begin{array}{l}\text { Surgery }^{a} \\
(\mathrm{n}=63)\end{array}$ \\
\hline & Mean \pm SD & Mean \pm SD & Mean \pm SD & $\begin{array}{l}\text { Mean change from } \\
\text { baseline }(95 \% \mathrm{Cl})\end{array}$ & Mean \pm SD & $\begin{array}{l}\text { Mean change from } \\
\text { baseline }(95 \% \mathrm{Cl})\end{array}$ & Mean \pm SD & $\begin{array}{l}\text { Mean change from } \\
\text { baseline }(95 \% \mathrm{Cl})\end{array}$ & Mean \pm & $\begin{array}{l}\text { SD Mean change from } \\
\text { baseline }(95 \% \mathrm{Cl})\end{array}$ \\
\hline DQ Score ${ }^{b}$ & $41 \pm 19$ & $65 \pm 21$ & $12(23)$ & $-29(-40$ to -19$)$ & $5(13)$ & $-60(-66$ to -55$)$ & $18(27)$ & $-24(-34$ to -14$)$ & $4 \pm 11$ & $-62(-68$ to -56$)$ \\
\hline AS-20 General Function ${ }^{c}$ & $64 \pm 26$ & $61 \pm 21$ & $83(16)$ & $19(10$ to 28$)$ & $90(15)$ & 29 (24 to 34$)$ & $79(21)$ & 14 (5 to 23 ) & $92 \pm 14$ & 29 (24 to 35$)$ \\
\hline AS-20 Reading Function ${ }^{c}$ & $79 \pm 21$ & $67 \pm 24$ & $82(23)$ & $3(-5$ to 10$)$ & $87(20)$ & $20(15$ to 24$)$ & $84(18)$ & $7(-3$ to 18$)$ & $90 \pm 16$ & 22 (16 to 27 ) \\
\hline AS-20 Self-perception ${ }^{c}$ & $92 \pm 17$ & $85 \pm 20$ & $91(13)$ & $-1(-6$ to 5$)$ & $95(14)$ & $9(5$ to 12$)$ & $94(11)$ & $3(-4$ to 11$)$ & $97 \pm 7$ & $11(6$ to 15$)$ \\
\hline AS-20 Interaction ${ }^{c}$ & $95 \pm 10$ & $94 \pm 12$ & $95(10)$ & $0(-4$ to 3$)$ & $97(12)$ & $3(1$ to 5$)$ & $98(5)$ & $2(-1$ to 5$)$ & $99 \pm 3$ & $5(3$ to 7$)$ \\
\hline
\end{tabular}

$A S-20$, Adult Strabismus-20 questionnaire; $C l$, confidence interval; $D Q$, Diplopia Questionnaire; $S D$, standard deviation.

aNine participants received additional treatment between the 10-week and 12-month examinations. For these participants, data collected at 10 weeks were included in the table, but data collected at 12 months were excluded from the table.

${ }^{\circ} \mathrm{DQ}$ scores range from 0 to 100 , with 0 being best (no diplopia) and 100 being worst (diplopia in all gazes all the time); improvement is thus reflected as negative change.

${ }^{\mathrm{C}} \mathrm{AS}-20$ domain scores range from 0 to 100 , with 0 being the worst HRQOL and 100 being the best HRQOL; improvement is thus reflected as positive change. 
Table 6A. Treatment success by prism strategy Initiated at enrollment

\begin{tabular}{|c|c|c|c|}
\hline \multirow{2}{*}{ Criteria } & \multicolumn{3}{|c|}{ Type of prism prescribed at enrollment ${ }^{a}$} \\
\hline & Correcting & High relieving & Low relieving \\
\hline \multicolumn{4}{|l|}{ Success at distance ${ }^{b, c}$} \\
\hline 10 weeks & $6 / 9(67 \%)$ & $8 / 12(67 \%)$ & $8 / 9(89 \%)$ \\
\hline & $95 \% \mathrm{Cl}, 30 \%$ to $93 \%$ & $95 \% \mathrm{Cl}, 35 \%$ to $90 \%$ & $95 \% \mathrm{Cl}=52 \%$ to $100 \%$ \\
\hline 12 months & $4 / 10(40 \%)$ & $5 / 9(56 \%)$ & $3 / 8(38 \%)$ \\
\hline \multicolumn{4}{|l|}{ Success at both distance and reading ${ }^{b}$} \\
\hline 10 weeks & 6/9 (67\%) & $8 / 12(67 \%)$ & 8/9 (89\%) \\
\hline 12 months & $\begin{array}{l}95 \% \mathrm{Cl}, 30 \% \text { to } 93 \% \\
4 / 10(40 \%)\end{array}$ & $\begin{array}{l}95 \% \mathrm{Cl}, 35 \% \text { to } 90 \% \\
5 / 9(56 \%)\end{array}$ & $\begin{array}{c}95 \% \mathrm{Cl}=52 \% \text { to } 100 \% \\
3 \text { of } 8(38 \%)\end{array}$ \\
\hline & $95 \% \mathrm{Cl}, 12 \%$ to $74 \%$ & $95 \% \mathrm{Cl}, 21 \%$ to $86 \%$ & \\
\hline
\end{tabular}

$\mathrm{Cl}$, confidence interval.

${ }^{a}$ The type of prism prescribed at enrollment was defined as percent of the distance deviation measured at enrollment, by prism and alternate cover test, corrected with prism: correcting prism ( $\geq 100 \%$ of distance deviation corrected with prism), high-relieving prism $(60 \%$ to $<100 \%)$, and low-relieving prism $(0 \%$ to $<60 \%)$.

'Definition of success: report on Diplopia Questionnaire of "rarely" or "never" diplopia over the preceding week and no alternative intervention.

'Two participants in correcting prism and 3 in low-relieving prism met failure criteria at 12 months because prism was increased between the 10-week and 12-month examination. One participant in high-relieving prism met failure criteria at 12 months despite not completing the 12-month visit because surgery was prescribed at the 10-week examination. 
Table 6B. Secondary clinical outcomes by type of prism treatment initiated at enrollment ${ }^{\mathrm{a}}$

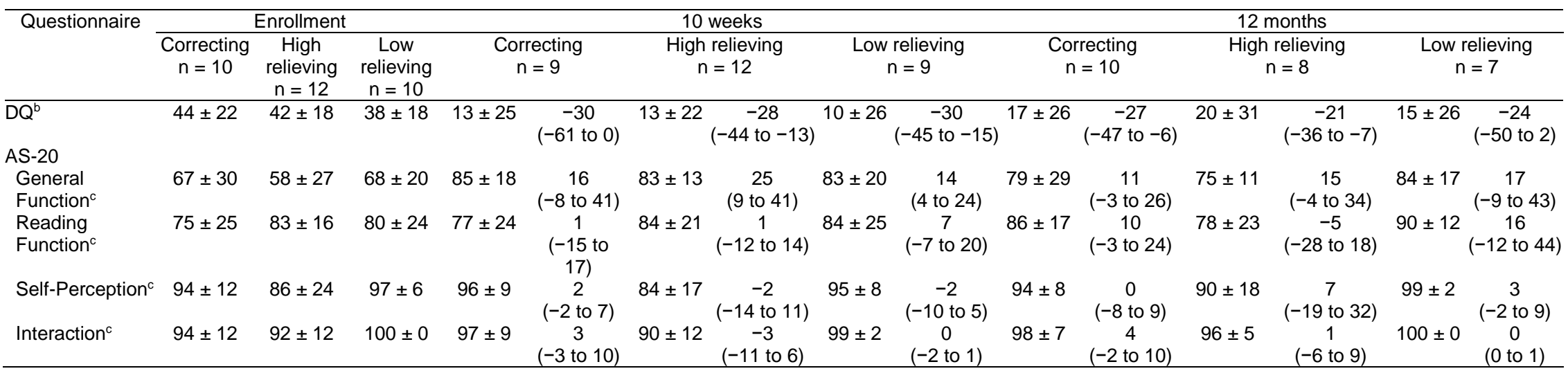

$C l$, confidence interval; $H R Q O L$, health-related quality of life; $S D$, standard deviation.

ascoring results shown as either mean \pm SD or mean change from baseline $(95 \% \mathrm{Cl})$

biplopia Questionnaire (DQ) scores range from 0 to 100, with 0 being best (no diplopia) and 100 being worst (diplopia in all gazes all the time); improvement is thus reflected as negative change.

'Adult Strabismus-20 questionnaire domain scores range from 0 to 100 , with 0 being the worst HRQOL and 100 being the best HRQOL; improvement is thus reflected as positive change. 
Table 7A. Treatment success by type of surgery

\begin{tabular}{lcc}
\hline & \multicolumn{2}{c}{ Type of surgery $^{\mathrm{a}}$} \\
\cline { 2 - 3 } & \multicolumn{2}{c}{ Bilateral LR resections $^{\text {Bilateral MR recessions }}$} \\
\hline Success at distance straight-ahead gaze \\
10 weeks & $37 / 40(93 \%)$ & $10 / 10(100 \%)$ \\
& $95 \% \mathrm{Cl}, 80 \%$ to $98 \%$ & $95 \% \mathrm{Cl}, 69 \%$ to $100 \%$ \\
12 months $^{\mathrm{c}}$ & $31 / 39(79 \%)$ & $9 / 10(90 \%)$ \\
& $95 \% \mathrm{Cl}, 64 \%$ to $91 \%$ & $95 \% \mathrm{Cl}, 56 \%$ to $100 \%$ \\
Success at both distance straight-ahead and reading gazes & & $10 / 10(100 \%)$ \\
10 weeks $^{\mathrm{b}}$ & $35 / 40(88 \%)$ & $95 \% \mathrm{Cl}, 69 \%$ to $100 \%$ \\
12 months $^{c}$ & $95 \% \mathrm{Cl}, 73 \%$ to $96 \%$ & $9 / 10(90 \%)$ \\
& $30 / 39(77 \%)$ & $95 \% \mathrm{Cl}, 56 \%$ to $100 \%$ \\
\hline
\end{tabular}

$C l$, confidence interval; $L R$, lateral rectus muscle; $M R$, medial rectus muscle.

aSuccess by other types of surgery not given because of small sample sizes: single MR recession (7); MR recession, LR resection (7); single LR tuck/plication (5); bilateral LR resection with superior transposition (1); bilateral MR recession with superior transposition (1); bilateral MR recession, superior rectus muscle nasal pole recession (1); bilateral MR recession, superior rectus muscle recession (1); LR tuck/plication, superior rectus muscle recession (1).

'Definition of success: report on Diplopia Questionnaire of "rarely" or "never" diplopia over the preceding week and no alternative intervention.

'Six participants enrolled in the surgical cohort (bilateral MR recession) met failure criteria at 12 months because additional treatment was prescribed at or after the 10-week examination but prior to the 12-month examination; 5 were prescribed prism glasses, and 1 received surgery. 
Table 7B. Secondary clinical outcomes by type of surgerya

\begin{tabular}{|c|c|c|c|c|c|c|c|c|c|c|}
\hline \multirow[t]{2}{*}{ Questionnaire } & \multicolumn{2}{|c|}{ Enrollment } & \multicolumn{4}{|c|}{10 Weeks } & \multicolumn{4}{|c|}{12 Months } \\
\hline & $\begin{array}{l}\text { Bilateral MR recessions } \\
\qquad(\mathrm{n}=41)\end{array}$ & $\begin{array}{l}\text { Bilateral LR resections } \\
(\mathrm{n}=10)\end{array}$ & Bilater & $\begin{array}{l}\text { I MR recessions } \\
(\mathrm{n}=40)\end{array}$ & Bilat & $\begin{array}{l}\text { al LR resections } \\
(\mathrm{n}=10)\end{array}$ & Bilatera & $\begin{array}{l}\text { al MR recessions }{ }^{\mathrm{b}} \\
(\mathrm{n}=34)\end{array}$ & Bilater & $\begin{array}{l}\text { al LR resections } \\
(\mathrm{n}=10)\end{array}$ \\
\hline $\begin{array}{l}\mathrm{DQ}^{\mathrm{c}} \\
\mathrm{AS}-20^{\mathrm{d}}\end{array}$ & $67 \pm 22$ & $75 \pm 19$ & $6 \pm 14$ & $-61(-69$ to -52$)$ & $1 \pm 3$ & $-74(-87$ to -61$)$ & $4 \pm 12$ & $-62(-72$ to -53$)$ & $3 \pm 9$ & $-72(-84$ to -60$)$ \\
\hline General Function & $60 \pm 24$ & $72 \pm 16$ & $89 \pm 18$ & 28 (20 to 35$)$ & $96 \pm 7$ & 24 (11 to 37 ) & $93 \pm 8$ & 30 (21 to 38$)$ & $98 \pm 4$ & 26 (15 to 36$)$ \\
\hline Reading $\mathrm{Fl}$ & & $68 \pm$ & $86 \pm 23$ & 18 & $85 \pm 21$ & & $93 \pm 10$ & 3 to 28 ) & $87 \pm 24$ & 30) \\
\hline Self-Perception & $82 \pm 24$ & $85 \pm 16$ & $95 \pm 16$ & $12(6$ to 18$)$ & $95 \pm 9$ & $10(-1$ to 21$)$ & $97 \pm 9$ & $13(7$ to 20$)$ & $99 \pm 4$ & 14 (3 to 24$)$ \\
\hline AS- $20^{d}$ & $92 \pm 15$ & $94 \pm 8$ & $96 \pm 15$ & $4(1$ to 7$)$ & $97 \pm 8$ & $3(-1$ to 7$)$ & $99 \pm 3$ & 6 (3 to 10$)$ & $100 \pm 0$ & $6(1$ to 12$)$ \\
\hline
\end{tabular}

$C l$, confidence interval; $L R$, lateral rectus muscle; $M R$, medial rectus muscle; $S D$, standard deviation.

aScore results shown as either mean \pm SD or mean change from baseline $(95 \% \mathrm{Cl})$.

bSix participants enrolled in the surgical cohort (bilateral MR recession) received additional treatment between the 10-week and 12-month examinations; for these participants, data collected at 10 weeks were included in the table, but data collected at 12 months were excluded.

'Diplopia Questionnaire scores range from 0 to 100, with 0 being best (no diplopia) and 100 being worst (diplopia in all gazes all the time); improvement is thus reflected as negative change.

${ }^{\mathrm{d}} \mathrm{AS}-20$ domain scores range from 0 to 100 , with 0 being the worst HRQOL and 100 being the best HRQOL; improvement is thus reflected as positive change. 
Table 8. Treatment success for participants prescribed surgery at enrollment by whether or not prism was prescribed prior to enrollment

\begin{tabular}{|c|c|c|}
\hline & $\begin{array}{c}\text { Treated with prism prior to } \\
\text { enrollment }\end{array}$ & $\begin{array}{c}\text { No prism treatment prior to } \\
\text { enrollment }\end{array}$ \\
\hline \multicolumn{3}{|c|}{ Success at distance straight-ahead gaze $^{a}$} \\
\hline \multirow[t]{2}{*}{10 weeks } & $50 / 55(91 \%)$ & $12 / 12(100 \%)$ \\
\hline & $95 \% \mathrm{Cl}, 80 \%$ to $97 \%$ & $95 \% \mathrm{Cl}, 74 \%$ to $100 \%$ \\
\hline \multirow{2}{*}{12 months $^{b}$} & $40 / 53(75 \%)$ & $10 / 12(83 \%)$ \\
\hline & $95 \% \mathrm{Cl}, 62 \%$ to $86 \%$ & $95 \% \mathrm{Cl}, 52 \%$ to $98 \%$ \\
\hline \\
\hline \multicolumn{2}{|c|}{$\begin{array}{l}\text { Success at both distance straight-ahead and reading gazes }{ }^{a} \\
10 \text { weeks }\end{array}$} & $11 / 12(92 \%)$ \\
\hline \multirow[b]{2}{*}{12 months $^{b}$} & $95 \% \mathrm{Cl}, 78 \%$ to $96 \%$ & $95 \% \mathrm{Cl}, 62 \%$ to $100 \%$ \\
\hline & $\begin{array}{l}39 / 53(74 \%) \\
95 \% \text { Cl. } 60 \% \text { to } 85 \%\end{array}$ & $\begin{array}{c}10 / 12(83 \%) \\
95 \% \text { Cl, } 52 \% \text { to } 98 \%\end{array}$ \\
\hline
\end{tabular}

$\mathrm{Cl}$, confidence interval.

aDefinition of success: report on Diplopia Questionnaire of "rarely" or "never" diplopia over the preceding week and no alternative intervention.

${ }^{\mathrm{b}}$ Eight treated with prism prior to enrollment and 1 with no history of prior treatment met failure criteria at 12 months because additional treatment was prescribed at or after the 10-week examination but prior to the 12-month examination; 8 were prescribed prism glasses, and 1 received surgery. 


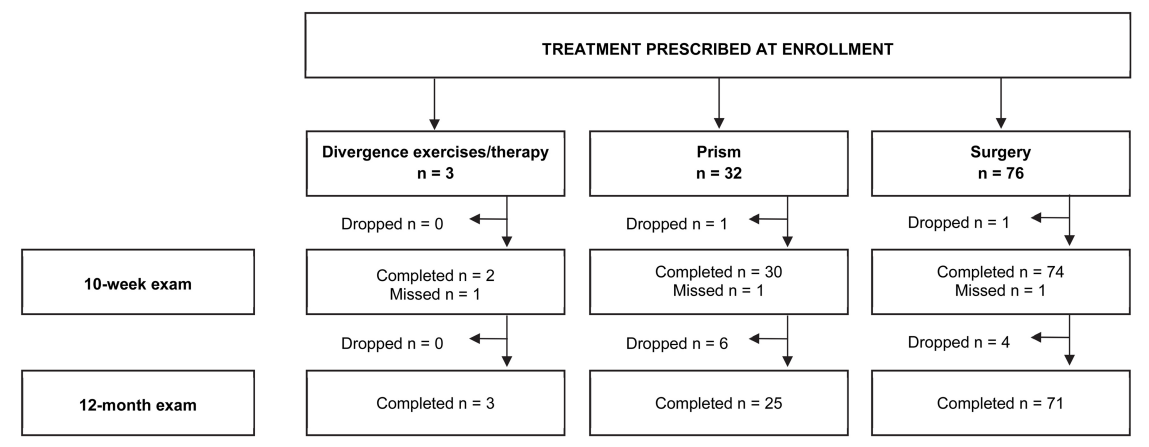

